\title{
CORRELAÇÕES ENTRE PARAMETROS DA ANÁLISE DE CRESCIMENTO DE CULTIVARES DE TOMATEIRO \\ (Lycopersicon esculentum Mill.) *
}

GILBERTO MARTINS**

EDMAR F.C. VASCONCELLOS $* * *$

ANTONIO A. LUCCHESI*****

\section{RESUMO}

No presente trabalho, estudaram-se as possiveis correlaçōes entre os parâme tros da anälise quantitativa de crescí mento vegetal, através dos dados de ex perimento conduzido com as cultivares de tomateiro, Kada, Angela, Floradel e Tropic, em sistema de cultivo para as condições do trópico-ümido, em Manaus, Amazonas, Brasil.

* Parte da dissertação de Mestrado apresentada pelo primeiro autor, à ESALQ/USP. Entregue para publicação em 28/08/85.

** Departamento de Agronomia, FUEL, Londrina, SP.

*** Departamento de Agricultura e Horticultura, E.S.A. "Luiz de Queiroz", USP.

**** Departamento de Botānica, E.S.A. "Luiz de Queiroz', USP. 
A partir da semeadura, a cada 14 dias, durante todo o ciclo do tomateiro, foram colhidas plantas e determinadas a altura, área foliar, peso da matéria seca da planta e peso da matéria fresca e seca dos frutos. Executou-se, dessa forma, a análise de crescimento vegetal dos cultivares e a correlação entre os parâmetros avaliados na planta: altura, produção, peso da matēria seca (PMS), indice de área foliar (IAF), taxa de produção de matëria seca (TPMS), taxa de produção de matéria seca vegetativa (TPMSV), taxa de produ ção de matéria seca dos frutos (TPMSF) taxa de crescimento relativo (TCR) e taxa de assimi lação líquida (TAL).

No estudo das correlações entre os parâmetros, a altura foi o que apresen tou maior quantidade de altas correlações, sendo assim, um bom indice para se estimar o desenvolvimento da planta. Existem correlações positivas: a) da altura com o IAF, com a produção, com o PMS e com a TPMS, ou seja, esses parāmetros estão diretamente correla cionados com a altura; b) do PMS com a produção e com o IAF. A TAL é mais afetada pela TCR, jà que apresentou to dos os coeficientes de correlação com valores baixos, e dentre eles, o mais elevado foi com a TCR. A TCR só teve altas correlações com a altura e o IAF, sendo essas correlaçōes negativas, ou seja, à medida que a planta cresce, di minui a TCR. Os cultivares mais produ tivos apresentaram altas correlações positivas entre TMSF e TPMS, mostrando 
ser a produtividade económica diretamente afetada pela produtividade bioló gica nesses cultivares.

INTRODUCEOO

o tomateiro é cultivado em todo o mundo, em condições de campo ou casa-de-vegetação, consumido "in na tura" ou industrializado, sendo citado por SONNENBERG (1979), como a hortaliça de maior expressão económica em muitos países.

Um vegetal de interesse econōmico, como uma plan ta de cultivo anual em crescimento, no caso, o tomate $\bar{i}$ ro, apresenta diferentes fases. No início como depeñ de de reservas contidas na semente, o crescimento é lento; posteriormente, após o desenvolvimento do sistema radicular e a emergência das folhas, tem um rápido crescimento atravēs da retirada de ägua e nutrien tes do substrato onde està e através da sua atividade fotossintética. Após atingir o tamanho definitivo, en tra para a fase de senescéncia (LUCCHESI, 1984).

Para tanto, o crescimento da planta pode ser medido pela obtenção de sua altura, mas, através da utilização da análi se quantitativa de crescimento, obtēm- se dados mais precisos e um nümero muito maior de informaçöes (LUCCHESI e MINAMI, 1980).

Segundo MAGALHÃES (1979), a anālise do crescimen to destina-se à avali ação da produção líquida das plañ tas, derivada do processo fotossintético, e é o resul= tado do desempenho do sistema assimilatório durante um certo período de tempo. Acompanhando assim a dinâmica da produção fotossintética, avaliada através da acumu- 
lação de matēria seca entre duas amostras sucessivas. O método é de grande valor na avaliação das diferenças intervarietais e interespecificas das diversas caracteristicas que definem a capacidade produtiva da planta, sendo usado para investigação do efeito de fenômenos ecológicos sobre o crescimento como adaptabilidade de es pécies em ecossistemas diversos, efeitos de competição, diferenças genotipicas da capacidade produtiva, influên cia de práticas agronônicas, além de fatores intrinse cos que estão associados com a fisiologia da planta.

A utilização dos parâmetros de crescimento vegetal, como um tipo de análise, teve início no começo do século com fitofisiologistas como BLACKMAN (1919) e BRIGS alii (1920), passando então a ser usado como mé todo básico para se calcular a estimativa da produtivi= dade biológica das comunidades vegetais. E, segundo RADFORD (1967), esse método continuarà a ser usado particularmente quando se deseja uma forma precisa de avaliação do crescimento da planta, sendo basicamente requeridas duas medidas periödicas para execução de uma simples análise de crescimento: o peso seco e a área foliar da planta.

Apesar de sua importância, è relativamente pequeno o nümero de trabalhos nessa área destacando-se alguns pesquisadores que estudaram a produtividade vege tal e o crescimento de värias culturas econômicas, utilizando-se dos parāmetros da anālise de crescimento. Co mo exemplo temos os trabalhos de RESS (1963) em dendé, MURAMOTO et alii (1965) em algodāo, KOLLER et alii(970) em feijão, FERREIRA (1972) em alho, MINAMI (1977) em be rinjela,CASTRO(1980) em soja e LUCCHESI (1980) em moran go, sendo que WALLACE e MUNGER (1965) e BUTTERY e BUZZEL (1972) utilizaram esses parâmetros para estudar o comportamento de diferentes cultivares de feijão e soja, respectivamente.

A técnica de análise de crescimento pode ser empregada mais efjcientemente em plantas de crescimento 
räpido, com folhas de forma regular e ciclo relativamen te longo como é o caso do tomateiro.

HEATH e GREGORY (1938) encontraram que a taxa de assimilação liquida (TAL) em tomateiro na fase vegetati va foi de $0,614 \mathrm{~g} / \mathrm{dm}^{2} /$ semana com fotoperíodo de $14 \mathrm{ho}=$ ras; 0,468 e 0,421 com fotoperíodo de $12,5 \mathrm{~h}$. e 10,5h, respectivamente. Já GOODALL (1945) cita valores médios de $0,481 \mathrm{~g} / \mathrm{dm}^{2} /$ semana para a TAL na cultura de veräo e de $0,117 \mathrm{~g} / \mathrm{dm}^{2} /$ semana na cultura de inverno, havendo al ta correlação entre TAL e intensidade luminosa, temperá tura diurna e noturna, fotoperiodo e umidade relativa. Comparando 2 sistemas de cultivo, BLACKMAN e WILSON (1951a) encontraram os seguintes resultados quanto à TAL e TCR (taxa de crescimento relativo):

a) Campo aberto: $\mathrm{TAL}=0,350 \mathrm{~g} / \mathrm{dm}^{2} /$ semana $e$ $T C R=0,133 \mathrm{~g} / \mathrm{g} / \mathrm{dia}$

b) Casa-de-vegetação: $T A L=0,513 \mathrm{~g} / \mathrm{dm}^{2} /$ semana e $T C R=0,199 \mathrm{~g} / \mathrm{g} / \mathrm{dia}$

Em nossas condições, CASTRo (1976) estudando os parāmetros da anālise de crescimento do tomateiro 'Miguel Pereira', encontrou na fase dos 40 aos 54 dias após o transplante a TAL de $0,102 \mathrm{~g} / \mathrm{dm}^{2} / \mathrm{dia}$ e a TCR de $0,133 \mathrm{~g} / \mathrm{dia}$.

Outros parāmetros de crescimento são utilizados nos estudos executados por WENT (1945) que compara dife rentes cultivares de tomateiro, baseando-se no peso da raiz, da parte aérea e do fruto, e também na altura do caule. Baseado na altura e peso da matéria seca GARGAN TINI e BLANCO (1963) encontraram um crescimento lentō do tomateiro do grupo Santa Cruz até os 40 dias, sendo dos 40 aos 100-120 dias a fase de maior crescimento, de acordo com a Tabela 1 . 
Tabela 1 - Crescimento do tomateiro do grupo Santa Cruz (GARGANTINI e BLANCO, 1963).

\begin{tabular}{|c|c|c|c|c|c|c|c|}
\hline \multirow{2}{*}{ PARAMETRO } & \multicolumn{7}{|c|}{ IDADE__d } \\
\hline & 20 & 40 & 60 & 80 & 100 & 120 & 140 \\
\hline Altura $(\mathrm{cm})$ & 9 & 25 & 52 & 90 & 137 & 120 & 125 \\
\hline
\end{tabular}

Peso da MS:

Veget at iva $\quad 0,04 \quad 2,12 \quad 11,62 \quad 50,40 \quad 90,3 \quad 92,6 \quad 69,5$ ( $g / p$ lanta)

Frutos

Total

$$
\begin{array}{rccrrrrr}
- & - & - & 4,80 & 46,7 & 104,0 & 130,5 \\
0,04 & 2,12 & 11,62 & 55,20 & 137,0 & 196,6 & 199,9
\end{array}
$$

FERNANDES et alii (1975) avaliando o crescimento do tomateiro do grupo Santa Cruz em cultura rasteira, confirmam dizendo que a fase de maior crescimento se dá no inicio da frutificação aos 60-70 dias de idade.

Comparando os cultivares Kada e Angela, BAUMGARTNER et alii (1976) encontraram os dados de altura e peso da matéria seca relatados na Tabela 2 .

Tabela 2 - Comportamento de diferentes cultivares de to mate em relação ao crescimento (BAUMGARTNER

\begin{tabular}{|c|c|c|c|c|c|c|}
\hline \multirow[t]{2}{*}{ CULTIVAR } & \multicolumn{4}{|c|}{ ALTURA $(\mathrm{cm})$} & \multicolumn{2}{|c|}{$\begin{array}{l}\text { PESO DA MATERIA } \\
\text { SECA ( } / \mathrm{pl} \text { lant } a)\end{array}$} \\
\hline & 15dias & 22dias & 29dias & 40dias & $30 \mathrm{dias}$ & $40 \mathrm{dias}$ \\
\hline $\begin{array}{l}\text { Kada } \\
\text { Ange la }\end{array}$ & $\begin{array}{l}21,4 \\
21,8\end{array}$ & $\begin{array}{l}33,9 \\
31,7\end{array}$ & $\begin{array}{l}50,7 \\
47,4\end{array}$ & $\begin{array}{c}70,3 \\
-\end{array}$ & $\begin{array}{l}2,5 \\
4,1\end{array}$ & 10,7 \\
\hline
\end{tabular}
et alii, 1976). 
Segundo HAGG et alii (1978), o cultivar Roma VF, de porte determinado, revelou um crescimento, expresso em produção de matéria seca, lento até aos 30 dias, porém, dobrando a cada quinzena, no período dos 45 aos 75 dias e atingindo o máximo aos 105 dias.

MATERIAL E METODOS

- trabalho consta de um estudo comparativo do com portamento de 4 cultivares de tomateiro realizado na Re gião Norte do Brasil, nas condiçōes da Amazônia. 0 experimento foi instalado no municipio de Manaus-Amazonas a $3^{\circ} \mathrm{S}$ de latitude, $59^{\circ} \mathrm{W}$ de longitude, no Instituto Ad ventista Agro-Industrial, localizado na rodovia AM-U10, $\mathrm{km} \mathrm{74,} \mathrm{no} \mathrm{período} \mathrm{de} 18 / 02 / 1981$, época da semeadura, até $10 / 06 / 1981$, quando foram coletados os ültimos dados. Nessa regiáo predominam os solos do tipo latossólico, principalmente o latossol amarelo, bastante intemperiza do, profundo, com baixa fertilidade e pH, porém, elevado teor de aluminio. o clima é tropical-úmido do tipo $\mathrm{Am}_{1}$, de elevada precipitação, temperatura e umidade relativa, com um pequeno periodo de estiagem leve.

0 experimento foi conduzido em instalação adequada à região e em implantação comercial, isto é, casa-de - vegetação aberta lateralmente, onde as plantas são cuI tivadas em caixas suspensas.

0 delineamento experimental utilizado foi $o$ de blocos casualizados, com parcela subdividida no tempo. o experimento constou de 4 tratamentos correspondendo as 4 cultivares mais recomendadas para esse sistema de cultivo, 2 do grupo Santa Cruz: Kada e Angela e 2 do grupo Salada: Floradel e Tropic, tendo 5 blocos e cons tituindo-se, portanto, em 20 parcelas. Cada parcela foi dividida em 8 sub-parcelas correspondentes às 8 épo 
cas de coletas de ados feitas a cada 14 dias a partir da semeadura, no decorrer do ciclo da cultura. Cada sub-parcela foi constituída por l caixa de cultivo com 8 plantas do mesmo cultivar, sendo 2 delas retiradas aleatoriamente para coleta de dados que forneceram os parâmetros da análise de crescimento a fim de avaliar qual a(s) melhor(es) cultivar(es) para esse sistema de cultivo.

A condução da cultura foi feita cuidadosamente, deixando 1 haste/planta, fazendo-se irrigação diaria mente, controle fitossanitário semanalmente, adubações foliares e todos os demais tratos culturais necessärios (tutoramento, desbrota, etc.).

Para avaliar o crescimento do tomateiro nesse sistema de cultivo e estudar o comportamento dos culti vares, utilizou-se os parâmetros da análise de cresci= mento. A partir da semeadura, a cada 14 dias, for am co tadas 2 plantas por sub-parcela e anotado os seguin tes dados:

- Altura da planta

- Area foliar

- Peso da matéria seca (PMS) da planta e dos frutos

- Peso da matéria fresca dos frutos (Produção).

Segundo recomendação de WILLIAMS (1946), BLACKMAN E WILSON (1951 a e b), WATSON (1952), RADFORD (1967), MAGALHÂES (1979) e LUCCHESI (1984), for am estu dados os seguintes parämetros da planta a fim de avaliar a cultivar:

- Indice de área foliar (IAF); Taxa de produção de matéria seca (TPMS); Taxa de crescimento relativo (TCR) e Taxa de assimilação líquida (TAL), sendo:

$$
I A F=\frac{A F}{S},
$$

onde : 


$$
\begin{aligned}
A F & =\text { ärea foliar da planta, em } \mathrm{cm}^{2} \\
S & =\text { ärea do solo disponível à planta, em } \mathrm{cm}^{2}
\end{aligned}
$$
planimetro.

A ārea foliar foi obtida atravēs do método do

$$
\text { TPMS }=\frac{P_{2}-P_{1}}{S\left(t_{2}-t_{1}\right)} \text {, em } g / m^{2} / d i a
$$

onde:

$$
\begin{aligned}
\mathrm{P}_{2} \text { e } \mathrm{P}_{1}= & \text { peso da matéria seca total }(\mathrm{g}) \text { da planta } \\
& \text { colhida na segunda e primeira amostragem }, \\
& \text { respectivamente. } \\
\mathrm{S}= & \text { ärea do solo disponivel à planta, em } \mathrm{m}^{2} \\
\mathrm{t}_{2} \text { e } \mathrm{t}_{1}= & \text { dias da segunda e primeira amostragem,res- } \\
& \text { pectivamente. }
\end{aligned}
$$

A TPMS foi desdobrada em taxa de produção da maté ria seca vegetativa (TPMSV), não sendo computado em $\overline{P_{2}}$ e $P_{1} O$ peso de matéria seca dos frutos, e a taxa de pro duçao de matéria seca dos frutos (TPMSF), onde $\mathrm{P}_{2}$ e $\mathrm{P}_{1}$ é o peso da matéria seca dos frutos somente.

$$
T C R=\frac{L P_{2}-L P_{1}}{t_{2}-t_{1}} \text {, em g/g/dia }
$$

onde:

$$
\begin{aligned}
\mathrm{L}= & \text { logaritro neperiano } \\
\mathrm{P}_{2} \text { e } \mathrm{P}_{1}= & \text { peso da matēria seca total }(\mathrm{g}) \text { da planta } \\
& \text { colhida na segunda e primeira amostragem, } \\
& \text { respectivamente. } \\
\mathrm{t}_{2} \text { e } \mathrm{t}_{1}= & \begin{array}{l}
\text { dias da segunda e primeira amostragem, res } \\
\text { pectivamente. }
\end{array}
\end{aligned}
$$




$$
T A L=\frac{P_{2}-P_{1}}{A_{2}-A_{1}} \times \frac{L A_{2}-L A_{1}}{t_{2}-t_{1}}, \text { em } g / \mathrm{dm}^{2} / \mathrm{dia}
$$

onde:

$$
\begin{aligned}
P_{2} \text { e } P_{1}= & \text { peso da matéria seca total }(g) \text { da planta } \\
& \text { colhida na segunda e primeira amostragem. } \\
& \text { respectivamente. }
\end{aligned}
$$
$\begin{aligned} A_{2} \text { e } A_{1}= & \text { área foliar da } p l a n f a \text { no tempo } t_{2} \text { e } t_{1} \text {, res } \\ & \text { pectivamente, em } d^{2} \text {. }\end{aligned}$

$L=$ logari tmo neperiano

$t_{2}$ e $t_{1}=$ dias da segunda e primeira amostragem, res pectivamente.

Alēm da anälise de variância utilizando o teste $F$ para comparar os cultivares, nos diferentes estádios da planta, e se obter as respectivas equaçöes de regressäo, foi tambëm feita uma análise de correlação múltipla entre os parāmetros estudados, dentro de cada cultivar Para cada correlação de interesse fez-se uma anālise de correlação linear com transformaçōes para se obter a me Ihor equação de regressão.

No estudo das correlações entre os parámetros uti lizados na análise quantitativa de crescimento vegetal, foram obtidas equaçōes de regressão para as correlaçōes mais altas e de maior interesse para cada cultivar.

1. Cultivar Kada 


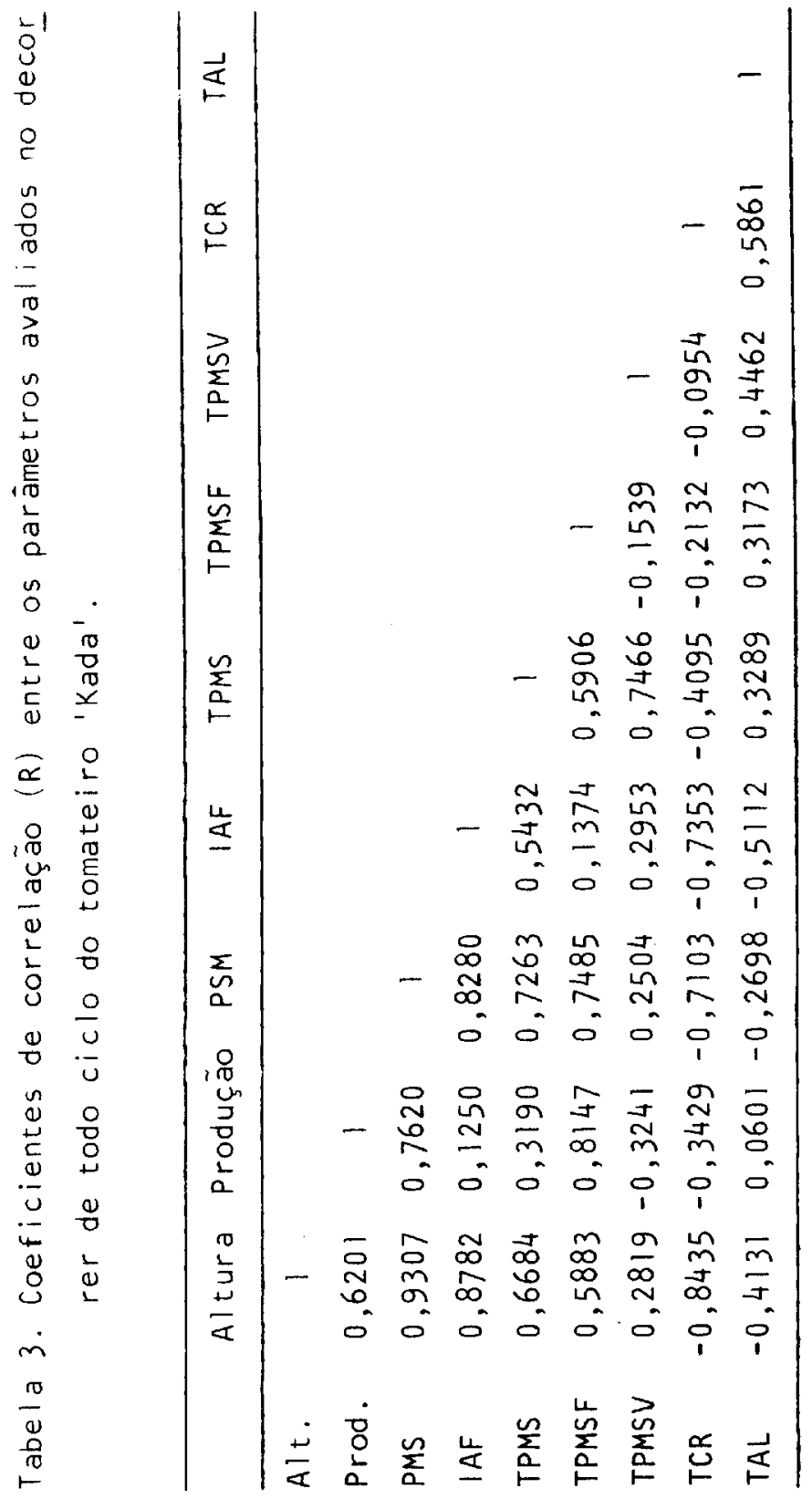


Tomando-se con base os maiores e mais importantes valores de $R$ acima de 0,8000 e que correspondem aos coeficientes de determinação $R^{2}$ acima de 0,6400 , ou seja, a porcentagem de variaçao explicada acima de $64,00 \%$, obteve-se as seguintes equações de correlação referentes ao cultivar Kada:

1.1. Altura $x$ PMS

$$
\operatorname{LnY}=-7,6902+2,3438 \operatorname{LnX} ; \quad R^{2}=0,98
$$

onde:

$$
\begin{aligned}
& \operatorname{Ln}=\text { logaritmo neperiano } \\
& Y=\text { peso da matéria seca da planta (PMS) em } \mathrm{g} \\
& X=\text { altura da planta em } \mathrm{cm} \\
& \text { 1.2. Altura } \times \text { IAF }
\end{aligned}
$$

$$
\frac{I}{Y}=-6,0023+\frac{686,5915}{X} ; \quad R^{2}=0,96
$$

onde:

$$
\begin{aligned}
& Y=\text { indice de área foliar da planta (IAF) } \\
& X=\text { altura da planta em } \mathrm{cm} \\
& \text { 1.3. Altura } \times \text { TCR } \\
& \quad Y=0,5872-0,1073 \mathrm{LnX} ; \quad R^{2}=0,84
\end{aligned}
$$

onde :

$$
\begin{aligned}
Y= & \text { taxa de crescimento relativo da planta (TCR) em } \\
& \mathrm{g} / \mathrm{g} / \mathrm{d} i \mathrm{a} \\
X= & \text { altura da planta em } \mathrm{cm} \\
L X= & \text { logaritmo neperiano }
\end{aligned}
$$


1.4. IAF $\times$ PMS

$$
\operatorname{Ln} Y=3,8560+1,6087 \operatorname{LnX} ; \quad R^{2}=0,97
$$

onde:

$$
\begin{aligned}
\mathrm{Ln} & =\text { logaritmo neperiano } \\
Y & =\text { peso da matéria seca da planta (PMS) em } \mathrm{g} \\
X & =\text { índice de área foliar (IAF) }
\end{aligned}
$$

2. Cultivar Angela

Tomando-se como base os mais importantes e maio res valores de $R$, acima de 0,8000 e que correspondem aos coeficientes de determinação $R^{2}$ acima de 0,6400 , ou seja, a porcentagem de variação explicada acima de $64,00 \%$, obteve-se as seguintes equaçōes de correlação, referentes ao cultivar Ángela:

2.1. Altura x Produção

$$
Y^{2}=(-179,4431+39,3890 \operatorname{Ln} x)^{2} ; \quad R^{2}=0,79
$$

onde:

$$
\begin{aligned}
& Y= \text { produção da planta em } \mathrm{g} \\
& X= \text { altura da planta em } \mathrm{cm} \\
& \operatorname{Ln}= \text { logaritıno neperiano } \\
& \text { 2.2. Altura } \times \text { PMS } \\
& \quad \operatorname{LnY}=-6,8626+2,1919 \operatorname{LnX} ; \quad \mathrm{R}^{2}=0,98
\end{aligned}
$$

onde:

$$
\text { Ln = logaritmo neperiano }
$$







$Y=$ peso da matēria seca da planta (PMS) em g

$X=$ altura da planta em $\mathrm{cm}$

2.3. Altura $\times$ IAF

$$
\frac{1}{Y}=-7,3578+\frac{781,7052}{X} ; \quad R^{2}=0,94
$$

onde:

$$
\begin{aligned}
& Y=\text { indice de àrea foliar (IAF) } \\
& X=\text { altura da planta em } \mathrm{cm} \\
& Y=(-19,7822+4,7388 \sqrt{x})^{2} ; R^{2}=0,93
\end{aligned}
$$

onde:

$$
\begin{aligned}
& Y=\text { produção da planta em } g \\
& X=\text { peso da matéria seca da planta (PMS) en } g \\
& \text { 2.5. } \frac{\text { IAF } \times \text { PMS }}{\quad} \\
& \quad \frac{1}{Y}=-0,1879+\frac{0,1700}{X} ; \quad R^{2}=0,98
\end{aligned}
$$

onde:

$$
\begin{aligned}
& Y=\text { peso da matéria seca da planta (PMS) em g } \\
& X=\text { índice de área foliar (IAF) }
\end{aligned}
$$

\section{Cultivar Floradel}

Tomando-se como base os mais importantes e maiores valores de $R$, acima de 0,8000 e que correspondem 
aos coeficientes det determinaçāo $R^{2}$, ou \% de variação explicada, acima de 0,6400 , ou $64,00 \%$, obteve-se as seguintes equaçōes de correlação referentes ao cultivar Florade I:

\subsection{Altura $\times$ Produção}

$$
\operatorname{LnY}=10,4670-\frac{633,6979}{x} ; \quad R^{2}=0,89
$$

onde :

$$
\begin{aligned}
\operatorname{Ln}= & \text { logaritmo neperiano } \\
Y= & \text { produção da planta em } \mathrm{g} \\
X= & \text { altura da planta em } \mathrm{cm} \\
\text { 3.2. Altura } \times \text { IAF } & \frac{45,1078}{X} ; \quad R^{2}=0,96
\end{aligned}
$$

onde:

$$
\begin{aligned}
\mathrm{Ln} & =\text { logaritmo neperiano } \\
Y & =\text { indice de ärea foliar (IAF) } \\
X & =\text { altura da planta em } \mathrm{cm}
\end{aligned}
$$

\subsection{Altura $\times$ TPMS}

$$
\operatorname{Ln} Y=2,2122-\frac{54,5424}{X} ; \quad R^{2}=0,94
$$

onde :

$$
\begin{aligned}
\mathrm{Ln}= & \text { logaritmo neperiano } \\
\mathrm{Y}= & \mathrm{t} \text { axa de produção de matëria seca da planta(TPMS) } \\
& \text { em } \mathrm{g} / \mathrm{m}^{2} / \mathrm{dia}
\end{aligned}
$$




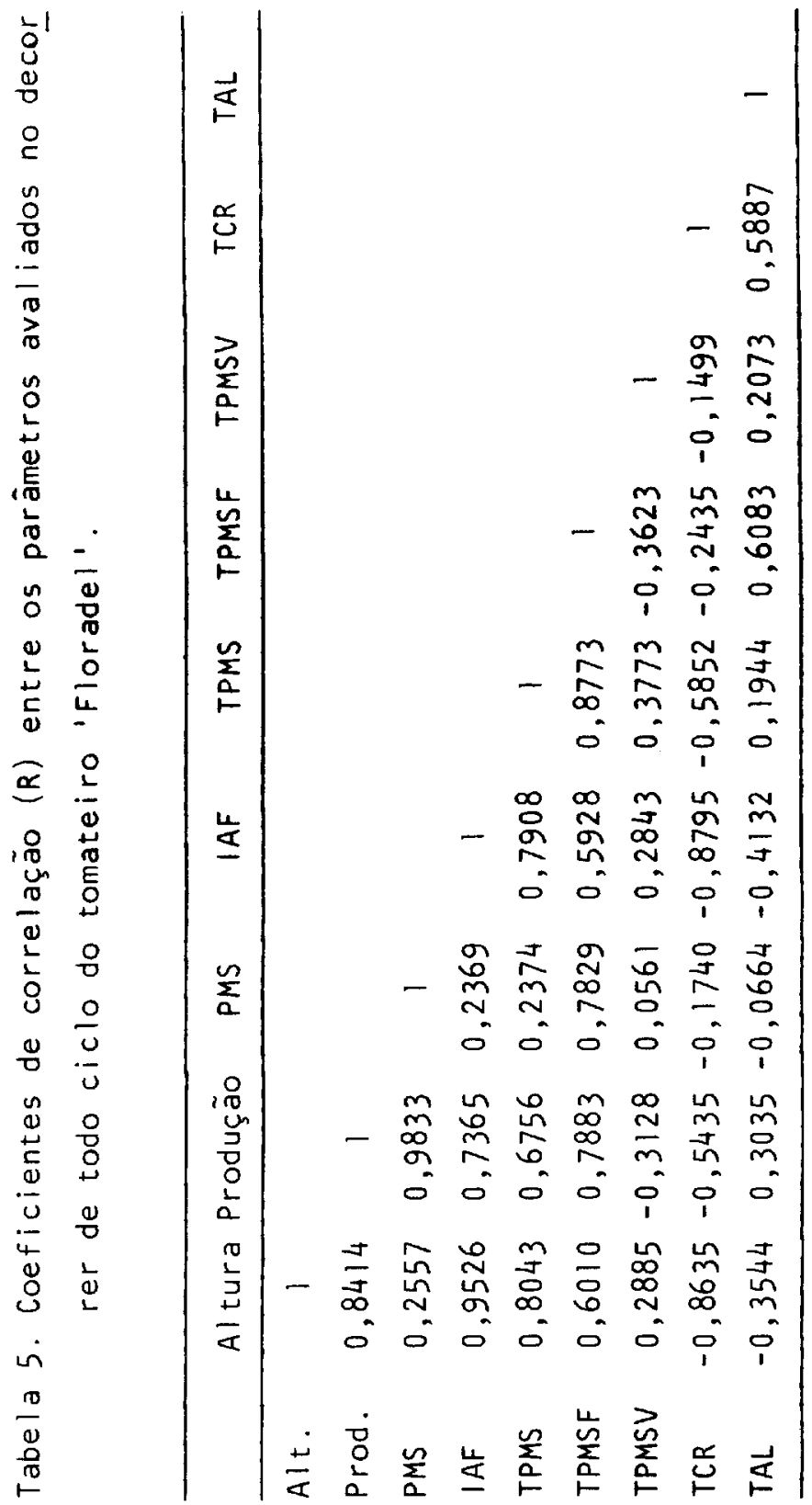


$X=$ altura da planta em $\mathrm{cm}$

3.4. Altura $\times$ TCR

$$
Y=0,4812-0,0880 \operatorname{Ln} X ; \quad R^{2}=0,88
$$

onde:

$$
\begin{aligned}
& Y=\text { taxa de crescimento relativo }(T C R) \mathrm{em} \mathrm{g} / \mathrm{g} / \mathrm{dia} \\
& L n=\text { logaritmo neperiano } \\
& X=\text { altura da planta em } \mathrm{cm} \\
& \text { 3.5. PMS } \times \text { Produção } \\
& \quad Y=(-15,4057+4,4577 \sqrt{\mathrm{X}})^{2} ; R^{2}=0,97
\end{aligned}
$$

onde:

$$
\begin{aligned}
& Y=\text { produçäo da planta em } g \\
& X=\text { peso da matëria seca da planta (MS) em } g \\
& \text { 3.6. } \frac{\text { IAF } X \text { TCR }}{\quad Y=0,06621-0,0694 \mathrm{LnX} ; \quad R^{2}=0,85}
\end{aligned}
$$

onde:

$$
\begin{aligned}
Y= & \text { taxa de crescimento relativo da planta (TCR) em } \\
& g / g / d i a \\
X= & \text { indice de área foliar } \\
L n= & \text { logaritmo neperiano }
\end{aligned}
$$

\section{Cultivar Tropic}

Tomando-se como base os mais importantes e maiores valores de $R$, acima de 0,800 , que correspondem aos 





coeficientes de determinaçào $R^{2}$, ou \% de variação expli cada, acima de 0,6400 ou $64,00 \%$, obteve-se as seguinte $\bar{s}$ equações de correlação, referentes ao cultivar 'Tropic':

4.1. Altura $x$ Produção

$$
\operatorname{Ln}=10,1152-\frac{534,7541}{x} ; \quad R^{2}=0,74
$$

onde:

$$
\begin{aligned}
& \text { Ln }=\text { logaritmo neperiano } \\
& Y=\text { produçáo da planta em } 9 \\
& X=\text { altura da planta em } \mathrm{cm} \\
& \text { 4.2. Altura } \times \text { PMS }
\end{aligned}
$$

$$
\operatorname{Ln} Y=7,1235+2,3467 \operatorname{Ln} X ; \quad R^{2}=0,97
$$

onde :

$$
\begin{aligned}
& \operatorname{Ln}=\text { logaritmo neperiano } \\
& Y=\text { peso da matéria seca da planta (PMS) em } \mathrm{g} \\
& X=\text { altura da planta em } \mathrm{cm} \\
& \text { 4.3. Altura } \times \text { IAF }
\end{aligned}
$$$$
\operatorname{LnY}=-7,0631+1,5399 \operatorname{LnX}, \quad R^{2}=0,96
$$

onde:

$$
\begin{aligned}
\mathrm{Ln} & =\text { logaritmo neperiano } \\
Y & =\text { indice de ärea foliar da planta (IAF) } \\
X & =\text { altura da planta em } \mathrm{cm}
\end{aligned}
$$


4.4. Altura $\times$ TCR

$$
Y=0,4872-0,0899 \operatorname{LnX} ; \quad R^{2}=0,85
$$

onde:

$$
\begin{aligned}
Y= & \text { taxa de crescimento relativo da planta (TCR) em } \\
& \mathrm{g} / \mathrm{g} / \mathrm{dia} \\
\mathrm{Ln}= & \text { logar itmo neperiano } \\
X= & \text { altura da planta em } \mathrm{cm} \\
\text { 4.5. } & \text { PMS } \times \text { Produçào } \\
& Y=117,4111+0,569 \mathrm{X}^{2} ; \quad \mathrm{R}^{2}=0,93
\end{aligned}
$$

onde:

$$
\begin{aligned}
& Y=\text { produção da planta em } g \\
& X=\text { peso da matéria seca da planta (PMS) en } g \\
& \text { 4.6. } \frac{\text { IAF XPMS }}{\operatorname{LnY}=3,6236+1,5009 \operatorname{LnX} ; \quad R^{2}=0,98}
\end{aligned}
$$

onde:

$$
\begin{aligned}
& \text { Ln }=\text { logaritmo neperiano } \\
& Y=\text { peso da matēria seca da planta (MS) em } g \\
& X=\text { indice de ärea foliar da planta (IAF) } \\
& \text { 4.7. } \frac{\text { IAF } x \text { TCR }}{Y=0,2768-9,1816 \sqrt{X} ; \quad R^{2}=0,83}
\end{aligned}
$$

onde: 


\section{$Y=$ taxa de crescimento relativo da planta (TCR) em $g / g / d i a$ \\ $X=$ indice de àrea foliar da planta (IAF)}

- estudo da correlação entre os parāmetros avaliados, nos diferentes estádios do ciclo do tomateiro, lapresentou certa semelhança nos 4 cultivares. Verificou-se que a altura foi o parāmetro que apresentou a maior quantidade de correlações altas $(R: 0,80)$, mostrando, assim, ser um bom índice para estimar o crescimento da planta.

As correlaçōes maiores e mais importantes foram:

1. Correlação negativa entre a altura e a TCR, sendo a única correlação negativa comum a todos os cultivares estudados, verificando-se que, à medida que a planta cresce, diminui a TCR, muito provavelmente devido ao efeito do auto-sombreamento. 0 mesmo fato foi tambëm constatado pela correlaçāo negativa entre TCR e IAF. Observou-se que a TCR sō apresentou correlação positiva, embora baixa, com a TAL. Sabe-se que uma planta com baixa TAL não é necessariamente, uma planta de baixa produtividade biológica; nesse aspecto, o IAF é mais importante. Isto foi constatado pela alta correlação entre PMS e IAF.

2. Correlações positivas de altura: com o lAF, em to dos os cultivares; com a produção, exceto no cultivar Kada; com o PMS, exceto no cultivar Floradel; e con a TPMS, somente no cultivar Floradel. Observou-se, em todos os cultivares, correlação positiva alta entre o PMS e a produção, exceto para o cultivar Kada, e entre - PMS e o IAF, exceto para o cultivar Floradel.

As altas correlações positivas da altura com o PMS e o IAF demonstraram que houve um bom crescimento do tomateiro nesse sistema de cultivo. 
A TAL apresentou todos os coeficientes de correlaçào comi valores baixos, e dentre eles, o mais elevado foi com a TCR. Caso semelhante foi observado com a TCR que apresentou, em relação aos demais parâmetros, poucas correlaçōes e quando elas ocorreram, foram nega tivas. Este fato poderia ser melhor entendido com base no conhecimento de que para os parämetros TCR $E$ TAL, o incremento de material produtivo está relaciona do com outro fator de crescimento da própria planta. como $n$ seu peso ou a sua área foliar. Estes paràmetros avaliam a capacidade de produção biológica da planta baseada naquilo que jà está formado.

0 desdobramento da TPMS em TFMSV e TPMSF demonstrou que no inicio a TPMS seria a própria TPMSV. Porém. na fase de frutificaçào a TPMSV tende a diminuir, jà que ocorre a translocação de boa parte do material sintetizado para os frutos, aumentando a TPMSF e resul tando num equilibrio na TPMS. Foram observadas, nos cultivares mais produtivos, correlaçöes positivas mais altas entre a produtividade biológica (TPMS) e a produ tividade econômica ou agrícola (TPMSF), sendo nos menos produtivos maior a correlação entre TPMS e TPMSV. Este fato evidencia ainda mais a maior capacidade produtiva dos cultivares Floradel e Tropic nesse sistema de cultivo.

\section{CONCLUSÕtS}

Nas condiçōes experimentais realizadas, chegou- se às seguintes conclusões:

1. A altura foi o parâmetro que apresentou maior quantidade de altas correlações sendo assim, um bom ín dice para se estimar o desenvolvimento da planta. 
2. Existem correlações positivas: a) da altura com o IAF, com a produção, com o PMS e com a TPMS, ou seja, esses parâmetros foram diretamente afetados pela altura; b) do PMS com a produção e com o laF.

3. A TAL è mais afetada pela TCR, já que apresentou todos os coeficientes de correlação com valores baixos, e dentre eles, o mais elevado foi com a TCR.

4. A TCR só teve altas correlaçōes com a altura e o IAF, sendo essas correlações negativas, ou seja, à medi da que a planta cresce, diminui a TCR.

5. Os cultivares mais produtivos apresentaram altas correlações positivas entre TPMSF e TPMS, mostrando ser a produtividade econômica, diretamente afetada pela pro dutividade biolögica, nesses cultivares.

\section{SUMMARY}

CORRELATIONS AMONG PARAMETERS OF GROWTH ANALYSIS OF TOMATO CULTIVARS (Lycopersicon esculentum MiII)

In the present research it has been studied the possible correlation among the parameters of quantitati ve analysis of vegetal growth through experimental data from tomato cultivars Kada, Angela, Floradel and Tropic carried out in the tropical-humid conditons of Manaus (Amazon State, Brazil) in a typical regional fashion (open side greenhouses with plants cultived in suspended wooden boxes).

Every 14 days plant samples were collected in the seedling time, to determine height, leaf area, dry matter, weight of the plant and fresh and dry matter weight of the fruits, during all the tomato cicle. Thus an analysis was made of the cultivar vegetal growth and 
the correlation among the evaluated plant parameters: height, yield, dry matter, weight (DMW), leaf area index (LAI), dry matter production rate (DMPR), vegetative dry matter production rate (VDMPR), fruit dry matter production rate (FDMPR), relative growth rate (RGR) and net assimilation rate (NAR).

Studying the correlation among the parameters, the height presented the greatest amount of hight correla tion, becoming this way a good cross-index to estimate the plant growth. The positive correlations are: a) Height with LAI, with yield, with DMW and with DMPR. that is, these parameters were directly correlationed with the height; b) OMW with yield and LAI. The NAR presented all coeficient correlations with low rates and among these rates the highest was with the RGR, showing this way to be affected by this parameter. The RGR only had high correlations with the height and the LAI, these correlations being negative, that is, while the plant grows, the RGR decreases. The most productive cultivars presented high positive correlations between FDWPR and DWPR, showing that the economic productivity is directly affected by the biological produc tivity in the cultivars.

\section{LTIFIRATURA GTIADA}

BAUMGARTNER, J.G.; HAGG, H.P.; OLIVEIRA, G.D.E PERECIN, D., 1976. Nutrição mineral de hortaliças XXVII. Toleräncia de cultivares de tomateiro (Lyeopersicum es eulentum Mill.) ao alumínio e ao manganés. Anais da ESALQ, Piracicaba 33:513-541.

BLACKMAN, V.R., 1919. The compound interest law and plant growth, Annals of Botany. 0xford 33:353-360 
BLACKMAN, G.E.E WILSUN, G.L., 1951a. Physiological and ecoiogical studies in the analysis of plant environment VII. Annais of Botany. Oxford 15(59):373408.

BLACKMAN, G.E.\& WILSON, G.L., 1951b. Physiological and ecological studies in the analysis of plant environment VI. Annals of Botany. Oxford 15(57):63-64.

BRIGS, G.E.; KIDD, F.E WEST, C., 1920. A quantitative analysis of plant growth 1 e $\mid 1$. Annals of Appljed Biology. Cambridge 7:103-123 e 202-223.

BUTTERY, B.R.; BUZZELL, R.1., 1972. Some differences between soybean cultivars observed by growth analy sis. Canadian Journal Plant Science. Ottawa 52:1320 .

CASTRO, P.R.C., 1976. Efeitos de reguladores de crescimento em tomatei ro (Lycopersicum esculentum Mill.). Piracicaba, ESALQ/USP, 148 p. (Tese de Doutoramento).

CASTRO, P.R.C., 1980. Efeitos de reguladores de cresci mento em soja |Glycine max (L.) Merril cv. Davis|. Piracicaba, ESALQ/USP, 174 p. (Tese de Livre-Docēncia).

FERNANDES, P.D.; CHURATA-MASCA, M.G.C.; OLIVEIRA, G. D. E HAAG, H.P., 1975. Nutriçăo mineral de hortaliças XXVII. Absorção de nutrientes pelo tomateiro (Lyoopersicum esculentur Mill.), em cultivo rasteiro. Anais da ESALQ. Piracicaba 32:595-608.

FERREIRA, F.A., 1972. Anälise do crescimento de quatro cultivares de alho (Al.Lium sativum L.). Viçosa, UFV, 41 p. (Dissertação de Mestrado).

GARGANTINI, H. E BLANCO, H.G., 1963. Marcha de absorção de nutrientes pelo tomateiro. Bragantia. Campi nas $22(56): 693-714$. 
GOODALL, D.W., 1945. The distribution of weight change in the young tomato plant. Annals of Botany. New York 9(34): 101-139.

HAAG, H.P.; OLIVEIRA, G.D.; BARBOSA. V. \& SILVA NETO, J. M., 1978. Nutrição mineral de hortaliças XXXIl. Marcha de absorção dos nutrientes pelo tomateiro ((Lycopersicum esculentum Mill.), destinado ao processamento industrial. Anais da ESALQ. Piracicaba $35: 243-270$.

HEATH, 0.V.S. E GREGORY, F.G., 1938. The constancy of the mean net assimilation rate and its ecological im portance. Annals of Botany. Oxford 2:811-818.

KOLLER, H.R.; NYQUIST, W.E. \& CHORUSK, I.S., 1970.

Growth analysis of the soybean community. Crop Scien ce. Madison 10:407-412.

LUCCHESI, A.A., 1980. Influência de fito-reguladores no crescimento e na produtividade de morangueiro (Eragaria spp.), cultivares Campinas e Monte Alegre. Piracicaba, ESALQ/USP, $154 \mathrm{p}$. (Tese de Livre-Docến cia).

LUCCHESI, A.A. E MINAMI, K., 1980. Anālise quantitativa de crescimento vegetal em cultivares de moranguei ro (Fragaria spp.), sob a influência de fito-reguladores de crescimento. Anais da ESALQ. Piracicaba $37(2): 553-593$.

LUCCHESI, A.A., 1984. Utilização prática da anālise quantitativa de crescimento vegetal. Anais da ESALQ (entregue para publicação).

MAGALHÃES, A.C.N., 1979. Análise quantitativa de crescimento. In: FERRI, G., coord. Fisiologia Vegetal I. São Paulo. Editora da Universidade de São Paulo, p. $331-350$. 
MINAMI, K., 1977. Anālise de crescimento e densidade de população de Solanum melongena L. - berinjela,cul tivada em delineamento sistemático e convencional. Piracicaba. ESALQ/USP, 81 p. (Tese de Doutoramento).

MURAMOTO, H.; HESKETH, J. \& EL-SHARKAWY, M., 1965. Relationship among rate of leaf area development, photosynthetic rate, and rate of dry matter production among american cultivated cottons and other species. Crop Science, Madison 5(2):163.166.

RADFORO, P.J., 1967. Growth analysis formulae-their use and abuse. Crop Science. Madison 7(3):171-175.

REES, A.R., 1963. Relationship between crop growth rate and leaf area index in the oil palm. Nature London 197(4862):63-64.

SONNENBERG, P.E., 1979. Olericultura Especial - Ia. Parte. 2a. ed. Goiània, Universidade Federal de Goìàs, 171 p.

WALLACE, D.H. \& MUNGER, H.M., 1965. Studies of the physiological basis for yield differences. 1. Growth analysis of six dry bean varieties. Crop Science. Madison $5(4): 343-348$.

WATSON, D.J., 1952. The physiological basis of varia tion in yield. Advances on Agronomy, New York 4 : $101-145$.

WENT, F.W., 1945. Plant growth under controlled conditions. $V$. The relation between age, light, variety and thermoperiodicity of tomatoes. American Journal of Botany. New York 32(8):469-479.

WILLIAMS, R.F., 1946. The physiology of plant growth with special reference to the concept of net assimilation rate. Annals of Botany. 0xford 10(37):41-72 